\title{
Model Predictive Control and Dynamic Operability Studies in a Stirred Tank: Rapid Temperature Cycling for Crystallization
}

\author{
GENE A. BUNIN, FERNANDO V. LIMA, \\ CHRISTOS GEORGAKIS, AND \\ CHRISTOPHER M. HUNT
}

Department of Chemical and Biological Engineering and Systems Research Institute, Tufts University, Medford, Massachusetts, USA

\begin{abstract}
A stirred-tank reactor was built with the objective of rapid and accurate temperature control in the reaction vessel. A first-principles heat transfer model was developed for the jacketed batch system, with the jacket inlet temperature used to control the vessel temperature. A model predictive controller was implemented to follow a rapidly changing temperature profile that cycled between steep heating and cooling motifs, and it was tested experimentally at progressively shorter temperature cycles. For a water-solvent-water-jacket system, a cycle consisting of increasing and decreasing the temperature by $15^{\circ} \mathrm{C}$ over a period of 20 min was achieved in practice. The performance of the MPC controller was explained by calculating the dynamic operability characteristics of the process.
\end{abstract}

Keywords Control of crystal morphology; Dynamic operability; Model predictive control; Temperature cycling

\section{Introduction}

When dealing with crystalline products and materials, the shape of the crystal is an important factor that affects the product's processing characteristics and other application properties. To use a very common example from the pharmaceutical industry, crystals with relatively higher sphericity (i.e., rough spheres or cubes) are generally preferred over the less-desired rod or needle-shaped habits. This is due to the improved washing and filtering properties inherent in the more spherical shapes. In addition to the flow properties, characteristics such as dissolution rate, particle size, and bulk volume can also be improved by controlling the crystal shape.

Past work on shape control has mostly restricted itself to two specific methodologies (Yang et al., 2006). The first has involved the use of additional solvents and impurities in the growth process, thereby influencing, through the additives, the specific growth rates of the different crystal faces. The other strategy has been to produce different polymorphs of a crystal with varying morphologies and lattice structures, thus not only changing the shape of the crystal but its chemical buildup as well.

Address correspondence to Christos Georgakis, Science and Technology Center, Tufts University, 4 Colby St., Medford, MA 02155, USA. E-mail: christos.georgakis@tufts.edu 
However, some groups have recently studied the use of operating conditions, such as cooling rate, to manipulate the shape of a crystal. One study in particular has focused on the crystal habit of potassium dihydrogen phosphate (KDP) and how changes in the cooling profile resulted in crystals with different aspect ratios (Yang et al., 2006). Additionally, recent progress in the modeling of crystal growth (Zhang et al., 2006) and crystal dissolution mechanisms has postulated an interesting new approach, suggesting that it may be possible to change the shape of the crystal by running the growth and dissolution steps in cycles (Snyder and Doherty, 2007). The objective of the research done here stems from these two examples and seeks to construct a system where the operating conditions can be varied quickly and accurately, with the capability to use both fast and slow cooling/heating rates, as well as the capability to follow many different kinds of temperature trajectories and foster growth and dissolution in patterns. By using these proposed shape control techniques, the issue of manipulating a crystal's morphology may be broken down to a complex, but theoretically achievable, control problem. The growth and dissolution concept is used as an illustrative basis for what this problem may look like in practice.

As growth and dissolution are phenomena driven by supersaturation and undersaturation, respectively, the experimental method for controlling the two relies on controlling the concentration in relation to the crystal's solubility at a given temperature or other conditions. When the concentration is above the system's solubility value, there is a drive to take the solute out of solution and contribute to crystal growth. Likewise, when the solubility is below the solubility value, there is a driving force for the solute to move into the solution and thereby start dissolving the crystals. Since solubility is a function of temperature, a very popular method for controlling the relative distance from the solubility curve simply involves controlling the temperature. Another common method is to add either solvent to decrease concentration or anti-solvent to relatively increase it. Both approaches are effective, and while the latter is generally quicker, the temperature manipulation approach is easier to implement as no volume change needs to be accounted for in the control of the process, and the experimental setup required is usually simpler (i.e., a jacketed batch reactor). Fast and accurate control of supersaturation and undersaturation via temperature changes, with subsequent attempts at shape control, are the ultimate aims of this project. The current article deals almost exclusively with this temperature control system, although the future uses of this system can be shown as follows.

Take the well-studied and unfavorably shaped KDP crystal, with the cycling process proposed in Figure 1, and vary the temperature to foster growth and dissolution in sequence. Starting with a saturated and seeded solution, the temperature is lowered in path (a) to drive crystal growth and lower the solute concentration in the solution. After a certain point, the system is heated back up to its initial temperature along path (b) to promote dissolution. The temperature is then kept constant on path (c), dissolving the crystals until a point close to the initial solute concentration is reached. The area left of the line "SN" and right of line 2 on the graph represents the area where secondary nucleation ( $\mathrm{SN}$ ) takes place, causing the appearance of new crystals. For illustrative purposes, we hypothesize that the number of initial crystals remains constant and the cycle affects only the crystal shape, without crossing the secondary nucleation line.

In order to experimentally test the hypothesis that cycles of dissolution and growth affect crystal shape, several important things are needed. Mainly, a proper 


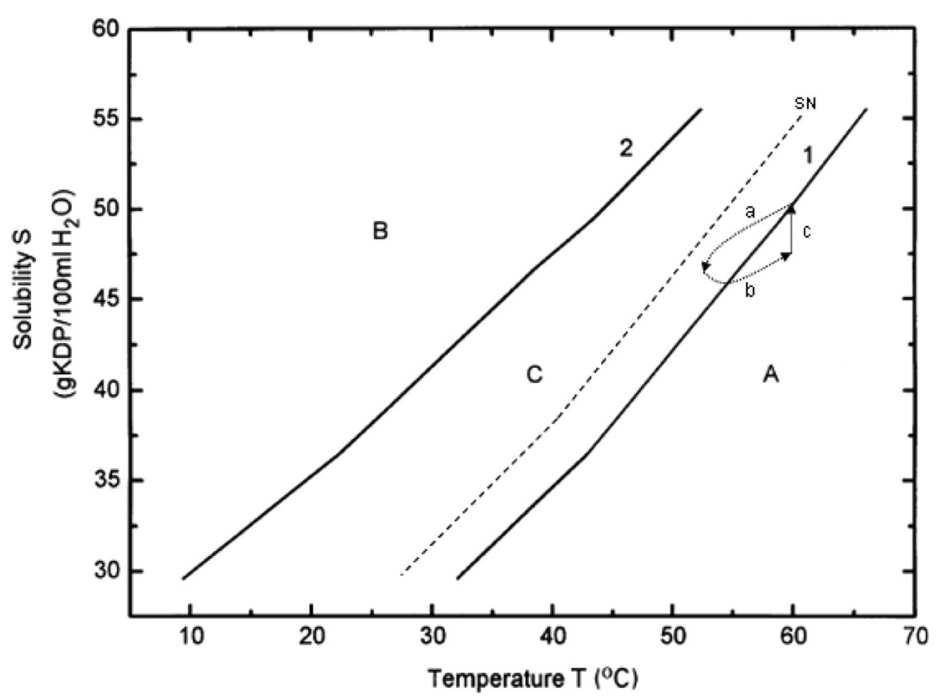

Figure 1. Proposed growth and dissolution cycling procedure for the KDP system. Key: 1, solubility curve; 2 , supersolubility curve; A, stable dissolution zone; B, unstable primary nucleation zone; $\mathrm{C}$, metastable zone (growth and secondary nucleation); SN, secondary nucleation (illustrative only, not based on any data). Solubility data and figure adapted from previous work (Shangfeng et al., 1999).

control system should be used so that the super/undersaturation can be effectively manipulated by changing the temperature of the solvent. While proportional-integral (PI) feedback has been successfully implemented for concentration control in the past (Zhou et al., 2006), it is unlikely that this type of controller could account for all of the hurdles present in crystallization processes. As mentioned in a recent review (Braatz, 2002), more advanced and robust control algorithms are needed, due to the significant challenges in controlling these processes, specifically the presence of disturbances and process constraints. Moreover, controlling the super/undersaturation in a crystallization process via temperature control implicitly requires the temperature to follow specific trajectories that are more complex than basic step changes. Model predictive control (MPC) is a widely used advanced model-based control algorithm that can cope with these challenges effectively (Qin and Badgwell, 2003) and thus was chosen here for the temperature control in the vessel. Since MPC is a model-based controller, a reasonably accurate process model is also required for effective implementation. In this work, a first-principles heat transfer model was developed to predict the temperature changes in the vessel. Finally, the construction of a fully automated system, needed to handle the challenging control algorithms, is also a necessity, and its setup is described here as well.

While very rapid temperature changes are not desired in some crystallization processes, recent work has shown that using relatively steep ramps in cooling some crystal systems has had beneficial effects on their crystal morphologies (Yang et al., 2006). For this reason, as well as for the purpose of designing a stirred-tank reactor system capable of working at several required operating conditions, the work here also aims to analyze how fast the temperature ramps can be achieved, in order to maximize the domain for experimental conditions in the future. Once certain process 
design limits are reached, not even the ideal feedback controller can make an inoperable process operate according to its desired set point trajectory, and so at that point the system operability must be examined. The final part of the work presented here thus deals with the dynamic operability of the system at hand and how its operability can be improved by using higher jacket inlet flow rates for temperature ranges that allow it.

This article is organized as follows. First, the experimental setup is described, then we briefly deal with how the first-principles heat transfer model was constructed. The model's implementation into the MPC controller is shown next, along with the simulated controller performance for varying cycling temperature profiles. In this same section, simulations for a more traditional feedback controller are performed in order to justify the use of the MPC controller in this system. Then we deal with the experimental temperature tracking in the crystallization vessel, and discuss the idea of dynamic operability, as well as the corresponding experimental results. Finally, conclusions are presented, followed by the proper acknowledgments.

\section{Experimental Setup}

A detailed control and flow schematic of the experimental setup is given in Figure 2, with a photograph of the working system provided in Figure 3. The utilized system was designed around a $3.0 \mathrm{~L}$ vessel, heated or cooled by a hot/cold water mixture sent through a $2.0 \mathrm{~L}$ jacket. The two hot and cold water feeds comprising the final mixture entering the jacket originate from a tap water source and from a NESLAB EX-220 temperature bath. The mixture outlet from the jacket is circulated back to the temperature bath, which has a manual overflow to the drain. In order to

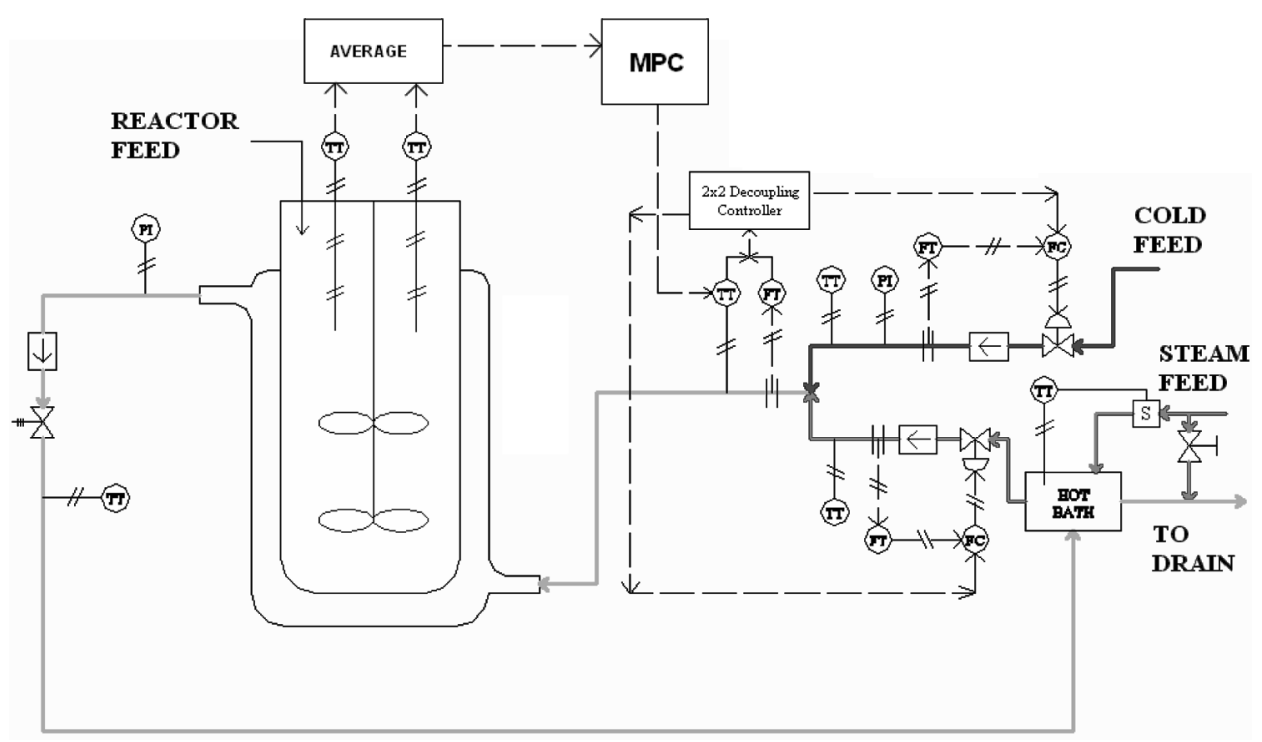

Figure 2. Flow and control schematic of the utilized system. Key: TT, temperature transducer; FT, flow transducer; FC, flow controller; PI, pressure indicator; S, solenoid valve. One-way valves are represented by arrowed blocks. Valve at the exit following the one-way valve is a pressure relief valve. 


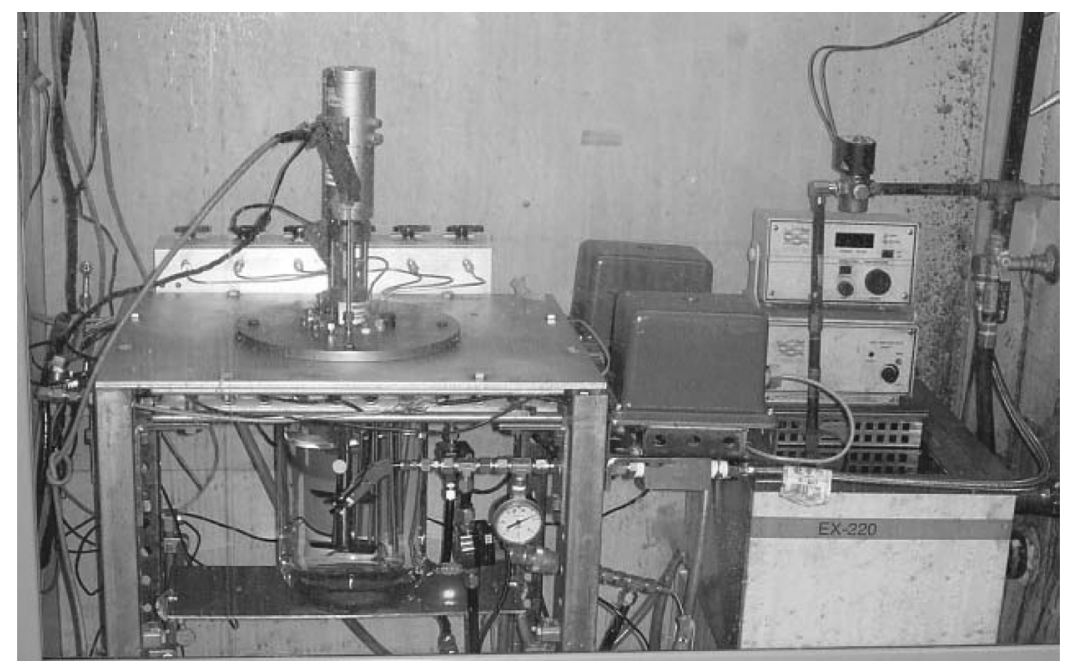

Figure 3. The working physical system.

maintain a reasonably hot feed temperature, a controlled flow of steam is fed into the bath via an on/off control mechanism, to keep the bath temperature at approximately $85^{\circ} \mathrm{C}$. The temperature of the cold tap water is assumed to be at around $20^{\circ} \mathrm{C}$ for both the simulation and experimental purposes. An Applikon ADI 1012 motor and speed controller is used to control the two stainless steel $45^{\circ}$ pitched impellers, allowing for mixing inside the main crystallizer tank at velocities ranging between 0 and 1250 RPM.

The designed control structure (Figure 2) consists of three layers, and borrows from the structure previously used by Miller and Rawlings (1994). In the innermost layer, two loops measure and control the flow rate of the cold and hot water streams. In the outermost layer, the MPC controller measures and controls the crystallizer temperature by manipulating the inlet temperature of the cooling/heating medium entering the jacket of the crystallizer. The flow rate of the cooling/heating medium into the jacket could have been used as a secondary manipulated variable in the MPC algorithm, but it is set to a constant value of $1.8 \mathrm{~L} / \mathrm{min}$ here (an exception is made, however, in the dynamic operability studies; see below). Selecting a fixed value for this flow rate linearizes the model that the MPC controller will use and has some implications on how fast the temperature can be changed over certain ranges. This is related to the dynamic operability of the process, as will be discussed later.

Between the inner and the outer control layers, there is a $2 \times 2$ decoupling structure in which the total incoming flow to the jacket is measured and controlled by the sum of the cold and hot flows. Similarly, the inlet jacket temperature is controlled by the ratio of the cold and hot stream flows. The two local controllers use a PI algorithm, tuned with the Ziegler-Nichols method, to manipulate the proportional valves (Omega PV 516-B) with a sampling time of $3 \mathrm{~s}$.

National Instruments' LabVIEW software was implemented for the control and data acquisition in the system. A total of eight thermocouples (Omega type-T) are used throughout the setup to collect temperature readings, seven of which are shown 
in Figure 2, with the eighth used to measure the ambient temperature around the crystallizer. Three flow meters (McMillan 104) are used to measure the flow rates of the hot, cold, and jacket inlet streams. A custom-built power supply board serves as the medium between the software and instrumentation. Voltages from the thermocouples and flow meters are relayed through the board and converted appropriately by LabVIEW's data acquisition software. Control signals from LabVIEW are designated in $\mathrm{mA}$ (4-20) for the proportional valves and are also relayed through the board. Finally, a solid state relay (Omega SSRL240) is utilized to deliver power to the solenoid valve (Omega SV241) controlling the steam, which operates on an on/off feedback mechanism with the thermocouple in the temperature bath. All the SISO (single-input-single-output) feedback control mechanisms, including the decoupler, are implemented via LabVIEW. The MPC algorithm is done in MATLAB (Mathworks, Inc.) and integrated into the LabVIEW code to interact with the cascade system via the MATLAB Script function.

\section{Model and Parameter Estimation}

Three critical areas of heat transfer were considered in the construction of the model for the temperature change inside the crystallizer, listed here in their descending order of importance:

(a) Between the crystallization medium, which will be referred to as the solvent, and the jacket.

(b) Between the jacket and ambient air in the lab.

(c) Between the solvent and the ambient air.

The solvent is assumed to be well mixed, so only a single energy balance is used to model the inside of the tank. The heating/cooling jacket is divided into two compartments for the model (analogous to two tanks in series), a bottom and a top with assumed equal volumes and heat transfer areas, thus requiring two additional energy balances. The approximation of equal volumes and equal heat transfer areas is close to reality, and does not introduce any significant error. The three ordinary differential equations (ODEs) governing the system are provided below:

$$
\begin{gathered}
\rho_{s} V_{s} C_{P, s} \frac{d T_{s}}{d t}=U_{j, s} A_{j, s}\left(T_{j 1}-T_{s}\right)+U_{j, s} A_{j, s}\left(T_{j 2}-T_{s}\right)+U_{s, r} A_{s, r}\left(T_{r}-T_{s}\right) \\
\rho_{j} V_{j 1} C_{P, j} \frac{d T_{j 1}}{d t}=\rho_{j} F_{j} C_{P, j}\left(T_{m i x}-T_{j 1}\right)+U_{j, s} A_{j, s}\left(T_{s}-T_{j 1}\right)+U_{j, r} A_{j, r}\left(T_{r}-T_{j 1}\right) \\
\rho_{j} V_{j 2} C_{P, j} \frac{d T_{j 2}}{d t}=\rho_{j} F_{j} C_{P, j}\left(T_{j 1}-T_{j 2}\right)+U_{j, s} A_{j, s}\left(T_{s}-T_{j 2}\right)+U_{j, r} A_{j, r}\left(T_{r}-T_{j 2}\right)
\end{gathered}
$$

where $\rho, V, C_{P}, F$, and $A$ denote density, volume, heat capacity, flow rate, and heat transfer area, respectively. The subscripts $s, j$, and $r$ refer to solvent, jacket, and room, respectively. The subscript mix refers to the jacket inlet, or mixture, stream. The subscript $j 1$ refers to the first jacket compartment (the bottom), and $j 2$ refers to the top. Finally, $U$ denotes the (overall) heat transfer coefficient, the parameter of greatest interest when constructing the model.

Although extensive work has been done on the development of intricate nonlinear models for this type of setup (Bequette et al., 2004), a linearized model 
was used here with the assumption that the nonlinearities present in this system were not sufficient to justify the development of a more complex model and that any minor model/process mismatch would be compensated for by the feedback capability of the MPC controller. The first main nonlinearity, the product of the flow rate and temperature as seen in Equations (2) and (3), was linearized by keeping the flow rate at a constant value of $1.8 \mathrm{~L} / \mathrm{min}$ throughout the course of the experiments and simulations (except during dynamic operability studies; see below). The other significant nonlinearity that is always present in a water-water energy transfer system is the viscosity of the water, which varies significantly as the temperature changes from 20 to $85^{\circ} \mathrm{C}$ and thus affects the heat transfer coefficient. This effect was also considered to be negligible, however, and the heat transfer coefficient terms were assumed constant over the relevant temperature ranges. Additionally, the stir rate inside the tank was maintained at a constant 300 RPM in order to eliminate heat transfer changes due to convection.

To calculate the values of the three heat transfer coefficients (jacket-solvent, jacket-room, solvent-room), the system model, comprised of (1)-(3), was implemented via MATLAB. Several experiments were run, in which water of varying temperatures was sent through the jacket to heat or cool a filled vessel, with all the temperature readings being recorded in the process. The resulting temperature profiles were fed into the model, and the heat transfer coefficients were calculated to minimize the least-squares error in the solvent and jacket temperatures (by MATLAB's subroutine fmincon). The model/process error generally fell under $0.5^{\circ} \mathrm{C}$.

\section{Temperature Control Simulation}

\section{Problem Definition}

To test the speed and accuracy of the temperature controller, a linear temperature cycling profile, consisting of a ramp temperature increase and decrease of $15^{\circ} \mathrm{C}$, was selected as the desired set point trajectory. This was done with the purpose of simulating the basic and fundamental characteristics that growth and dissolution cycling might possess. From a control perspective, this type of trajectory presents two challenges. The first is due to the fact that the set points are a series of ramps, rather than step changes, making it difficult for a standard PI controller to follow without any offset. The second, and bigger, challenge is due to the change in the sign of the slope that occurs at the end of each ramp. A traditional feedback controller would be at a disadvantage here, as the pure error-feedback mechanism could potentially achieve small or zero offset at the top/bottom of the ramp, especially if the time period was long enough but would fall behind on the turns. This is demonstrated later.

Before testing the MPC controller in the physical unit, simulations were done to validate its efficacy over a simpler controller, such as the proportional-integralintegral (PII). The PII, while a pure-feedback algorithm, has proven effective in the past for dealing with ramps in both set points and disturbances due to its second integrator action (Belanger and Luyben, 1997). All the simulations done here followed a similar scenario, with a starting steady-state jacket inlet temperature of $52.5^{\circ} \mathrm{C}$, midway between the theoretical mixture temperature constraints, $85^{\circ} \mathrm{C}$ for pure hot and $20^{\circ} \mathrm{C}$ for pure cold water streams. The corresponding steady-state value of the solvent temperature was approximately $51.5^{\circ} \mathrm{C}$, as predicted by the model. 
The controller was to follow the ramp set point, going $15^{\circ} \mathrm{C}$ up and $15^{\circ} \mathrm{C}$ down in $\tau_{p}$ minutes several times.

The period $\tau_{p}$ dictates the difficulty of the scenario, and ranges from $\tau_{p}=120 \mathrm{~min}$, for an easy scenario, to $\tau_{p}=10 \mathrm{~min}$, for an infeasible scenario for the specific unit due to dynamic operability limitations (see below). The high end in this range of periods was selected to represent the standard and slower ramps commonly used in industry, from where the period was then shortened to observe the decrease in controller performance, down to the inoperable case of $\tau_{p}=10 \mathrm{~min}$. As stated in the introduction, it is not desirable to change the temperature as quickly as $15^{\circ} \mathrm{C}$ in 5 or 10 min in some real crystallizations, but recent studies (Yang et al., 2006) have shown that cooling rates corresponding to the moderate-length periods (as fast as $0.4^{\circ} \mathrm{C} / \mathrm{min}$ ) could have benefits to the crystal shape. The case studies with the shortest periods are mainly performed here to test the control system's capabilities and the operability limitations of the current reactor system design.

All simulations were done with MATLAB. Details on the PII and MPC algorithms, as well as the simulation results for both controllers, are presented next.

\section{PII Algorithm}

The three-ODE fundamental model was expressed in terms of the proper deviation variables and was converted to the Laplace domain. The solvent and inlet jacket temperatures were defined as the output $(y)$ and input $(u)$, respectively. The derived transfer function expression, $g(s)$, is:

$$
\frac{y(s)}{u(s)}=g(s)=\frac{0.084 s+0.315}{s^{3}+3.993 s^{2}+4.157 s+0.326}
$$

with $s$ having units of $\min ^{-1}$. A transfer function for a PII controller with a single integral time squared to simplify the tuning procedure, per Belanger and Luyben (1997), was defined as:

$$
g_{c}(s)=K_{c}\left(1+\frac{1}{\tau_{I} S}\right)^{2}
$$

The standard closed-loop transfer function response is shown in Equation (6), with no significant disturbances or sensor dynamics assumed in the simulation:

$$
y(s)=\frac{g(s) g_{c}(s)}{1+g(s) g_{c}(s)} y_{d}(s)
$$

where $y$ represents the output, and $y_{d}$ represents the set point. The controller proportional gain $\left(K_{c}\right)$ and integral time constant $\left(\tau_{I}\right)$ parameters were tuned after extensive Routh array and root locus analysis to generate the results with the least simulated error, using the highest input values possible within the input constraints of $\pm 32.5^{\circ} \mathrm{C}$.

\section{MPC Algorithm}

The MPC formulation used here is based on previously developed approaches in the literature (Lundström et al., 1995; Garcia and Morshedi, 1986). For a SISO system, 
the state-space prediction model for the future output value $\left(y_{p}\right)$ is defined as (Lundström et al., 1995):

$$
y_{p}(k+1)=y(k)+a_{1} \Delta u(k)+d(k)
$$

where $y(k)$ denotes the output value at a current time $k$ in the absence of further control action, the scalar $a_{1}$ represents the first step response coefficient generated from the linear model of the plant, and $\Delta u(k)$ denotes the current change in the control action, which, when multiplied by $a_{1}$, yields the contribution of further control action to the output. Additionally, $y_{p}(k+1)$ denotes the future prediction at one time sample ahead, and $d(k)$ represents a current output bias correction, defined as the difference between the predicted output value and the plant measurement at time $k$. This correction is necessary, as some unmeasured disturbances and parameter uncertainties are usually unaccounted for by the model, generating a plant-model mismatch. Considering a prediction horizon of $n p$ samples, Equation (7) can be rewritten in the matrix form and expressed as:

$$
\mathbf{y}_{p}(k+1)=\mathbf{y}_{p}(k)+\mathbf{A} \Delta \mathbf{u}(k)+\mathbf{d}(k)
$$

where

$$
\begin{gathered}
\mathbf{y}_{p}(k+1)=\left[y_{p}(k+1), y_{p}(k+2), \ldots, y_{p}(k+n p)\right]^{T} \\
\mathbf{y}_{p}(k)=\left[y_{p}(k), y_{p}(k+1), \ldots, y_{p}(k+n p-1)\right]^{T} \\
\mathbf{A}=\left[\begin{array}{cccc}
a_{1} & 0 & \cdots & 0 \\
a_{2} & a_{1} & \cdots & 0 \\
\vdots & \vdots & \ddots & \vdots \\
a_{n p} & a_{n p-1} & \cdots & a_{1}
\end{array}\right] \\
\Delta \mathbf{u}(k)=[\Delta u(k), \Delta u(k+1), \ldots, \Delta u(k+n p-1)]^{T} \\
\mathbf{d}(k)=[d(k), d(k+1), \ldots, d(k+n p-1)]^{T} \\
\mathbf{y}_{p}(k) \in \mathbf{R}^{n p \times 1} ; \mathbf{y}_{p}(k+1) \in \mathbf{R}^{n p \times 1} ; \Delta u(k) \in \mathbf{R}^{n p \times 1} ; \mathbf{d}(k) \in \mathbf{R}^{n p \times 1} ; \mathbf{A} \in \mathbf{R}^{n p \times n p}
\end{gathered}
$$

with the matrix A representing the dynamic matrix, which includes all the first $n p$ step response coefficients $\left(a_{1}, a_{2}, \ldots, a_{n p}\right)$ obtained from the linear process model. Also, $y_{\mathrm{p}}(k)$ is initialized as the measured value from the plant at the application starting time $k$. For the purpose of this work, a constant output bias calculated at $k$ is propagated for the entire prediction horizon as defined in:

$$
d(k+l)=d(k) \text { for } l=1,2, \ldots, n p-1
$$

Moreover, the control action is calculated for only $m$ samples ahead (with $m<n p)$, allowing all the $\Delta \mathbf{u}(k)$ terms from $\Delta u(k+m)$ to $\Delta u(k+n p)$ to be set to 0 
and for $\Delta \mathbf{u}(k)$ to be truncated as in:

$$
\begin{aligned}
\Delta u(k+l) & =0 \quad \text { for } l=m, m+1, \ldots, n p \\
\Delta \mathbf{u}(k) & =[\Delta u(k), \Delta u(k+1), \ldots, \Delta u(k+m-1)]^{T}
\end{aligned}
$$

Finally, the MPC optimization problem of calculating the optimum $m$ moves for the inlet jacket temperature, so as to maintain the crystallizer temperature as close as possible to a specified reference trajectory, is solved by using the quadratic programming formulation of QDMC (quadratic programming solution of dynamic matrix control) (Garcia and Morshedi, 1986). Mathematically, this problem is stated as the minimization of the objective function $J$ in Equation (10) (the two-index notation has not used before Equation (10) for simplicity):

$$
\begin{aligned}
& J=\min _{\Delta \mathbf{u}(k \mid k)}\left\{\left\|\mathbf{W}\left[\mathbf{y}_{p}(k+1 \mid k)-\mathbf{y}_{s p}(k+1 \mid k)\right]\right\|^{2}+\|\mathbf{\Lambda} \mathbf{\Delta} \mathbf{u}(k \mid k)\|^{2}\right\} \\
& \mathbf{W} \in \mathbb{R}^{n p \times n p} ; \boldsymbol{\Lambda} \in \mathbb{R}^{m \times m}
\end{aligned}
$$

with $\mathbf{W}$ and $\boldsymbol{\Lambda}$ denoting the output weight and the input move suppression matrices, respectively, and $\mathbf{y}_{s p}$ denoting the set point trajectory values. Here the notation $(k+1 \mid k)$ represents the predicted value at $k+1$ calculated at a current time $k$. Once the calculation is done for the current time $k$, the first control action is implemented into the plant and the optimization problem is repeated at time $k+1$. All the other vectors are updated accordingly to properly simulate the plant response.

For the simulated cases in the next subsection, the sampling time is set at $1 \mathrm{~min}$. The inlet temperature constraints are specified as before and are incorporated into the quadratic programming formulation. For the specific purpose of comparison between a traditional feedback and an MPC controller, it is assumed that there is no plant-model mismatch, and so the vector $\mathbf{d}(k)$ is constantly set equal to 0 . Finally, the input move suppression and the output weight matrices are considered, throughout the simulations, to be diagonal with 0.1 and 1.0 (respectively) in their diagonals.

\section{Simulated Results for the PII and MPC}

For the cases where the ramp goes up/down $15^{\circ} \mathrm{C}$ in $\tau_{p}=120,90$, or $60 \mathrm{~min}$, nearly perfect performance is achieved by both the MPC and the PII controllers, with the former getting significantly less error, but qualitatively not enough to justify its implementation over the less sophisticated PII. For more ambitious cases, however, the fundamental differences between the two controllers become apparent. In the case where $\tau_{p}=30 \mathrm{~min}$, the PII cannot account for the sharp change in the set point ahead of time, and therefore suffers a significant lag on each turn, while the MPC controller makes each turn fairly well, as shown in Figure 4 . For $\tau_{p}=20 \mathrm{~min}$, the difference becomes even more apparent, as the PII exhibits significant lag, while the MPC is still capable of performing reasonably well (see Figure 5).

The MPC was therefore chosen over the traditional feedback control algorithm for the system at hand, due to its capability of achieving better performance for all simulated ramps, with exceedingly better performance for the more ambitious cases. This was largely expected, as experimental work comparing the performance of a 


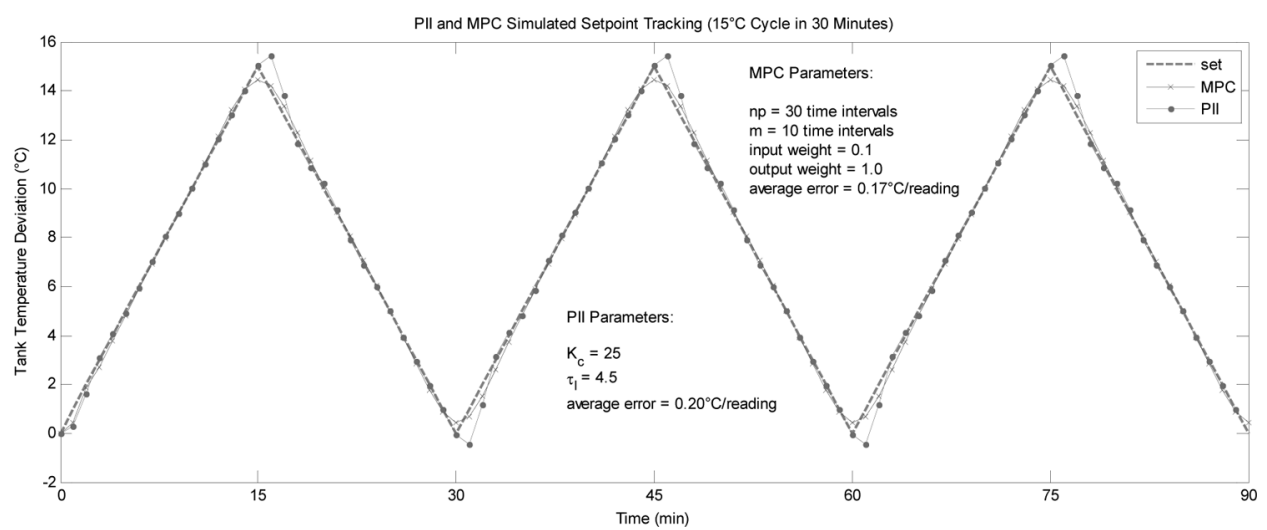

Figure 4. Simulated PII and MPC tracking of the ramp set point ("set") for the case of $\tau_{p}=30 \mathrm{~min}$. Here, the steady state of 0 corresponds to an absolute temperature of $51.5^{\circ} \mathrm{C}$. Control action intervals are $1 \mathrm{~min}$ for both controllers.

more traditional controller (in this case, a PI) and an MPC in crystallizer temperature tracking has been carried out before for longer time intervals (Shen et al., 1999), with the latter giving better results. In the study here, the focus is on the controller's ability to achieve fast heating/cooling, which may be desired in some instances of super/undersaturation control or in experiments where the cooling rate is varied, and so a controller that can achieve, as an example, a $15^{\circ} \mathrm{C}$ ramp accurately in only 10 min may be of significant benefit. Additionally, the ability of the MPC algorithm to include process constraints in its formulation provides yet another advantage over the unconstrained traditional feedback algorithm. Last, while the effect of unmeasured disturbances and parameter uncertainties was neglected in the simulations, it does become an issue in a real system, and the MPC's ability to predict the future effect of disturbance values in the plant and to take the necessary control action to compensate for them (over its defined prediction horizon) also make it superior to the PII algorithm.

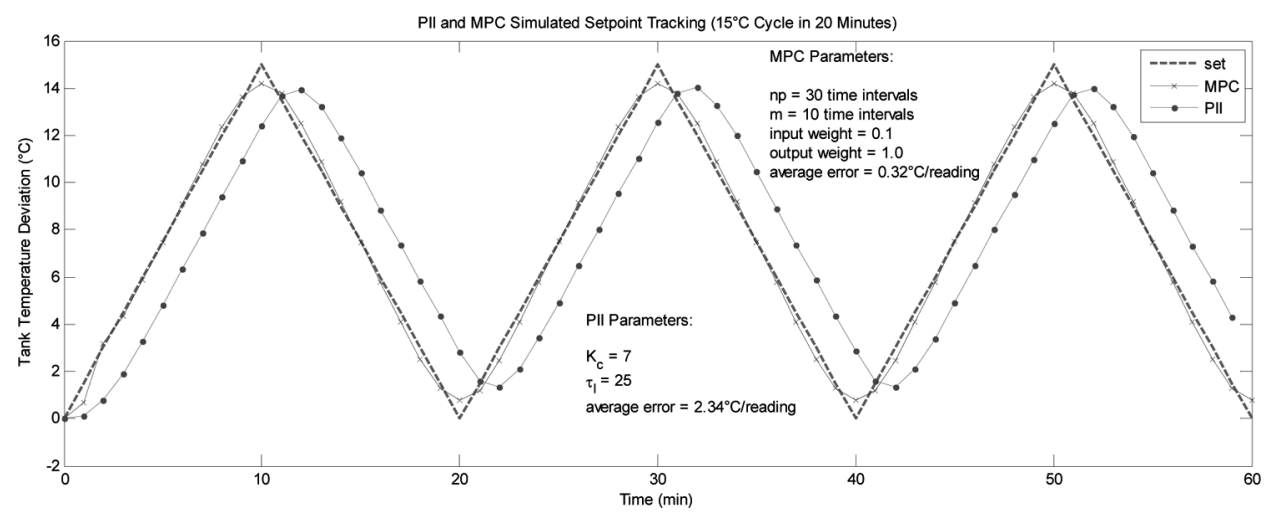

Figure 5. Simulated PII and MPC tracking of the ramp set point ("set") for $\tau_{p}=20 \mathrm{~min}$. 
Based on this list of justifications, an MPC controller was installed in the laboratory crystallizer via a MATLAB Script function in the LabVIEW software. As discussed in the previous section, the optimization problem was solved at each minute, and the optimal control action predicted by the MPC was implemented, changing the set point of the inlet jacket temperature for the cascaded PI system to achieve. The disturbance vector was estimated by measuring the difference between the predicted value and the crystallizer temperature reading each minute and propagating that value over the prediction horizon. The input move suppression and output weight matrices used were the same as in the simulations, with 0.1 and 1.0 values in the diagonals, respectively.

\section{Results and Discussion}

\section{Experimental Thermocycling}

The same scenarios, as described above, were carried out with the implemented MPC in the physical system, with $\tau_{p}$ again ranging from 10 to $120 \mathrm{~min}$. Accurate control was achieved for the slower cases. Errors of less than $0.5^{\circ} \mathrm{C} /$ reading were consistent for the $120,90,60$, and $30 \mathrm{~min}$ periods, but even with $\tau_{p}=20 \mathrm{~min}$, fairly good performance was seen, as shown in Figure 6(a).

A few points merit attention. As seen in both the simulations and in the experimental runs, the MPC controller never reached the top or bottom of each cycle, but rather cut across smoothly just under/over it. This was expected, as the discontinuity in the derivative of the set point trajectory becomes progressively harder for the system to approximate with decreasing $\tau_{p}$ values. Specifically, there is a compromise between achieving these turning points and minimizing the prediction error. Further tuning of the weights, or a varying weight scheme in the prediction horizon, may remediate this problem.

In addition, other factors can be taken into account, such as control action frequency and the implementation of a more detailed disturbance model to improve the general MPC accuracy. The MPC action sample interval of $1 \mathrm{~min}$, a common industry standard (Qin and Badgwell, 2003), was employed here, but a shorter sampling time may be required for faster temperature ramps. In the same way, more frequent action from the cascaded control system in the inner two layers would be of benefit as well, but this is currently a limitation of the utilized computer system's hardware. A general step disturbance model proved effective in the experimental runs, and additional runs done without any disturbance model showed a large decrease in performance, thus demonstrating the model's efficacy. However, the implementation of an optimal state observer, such as the Kalman filter (Lundström et al., 1995), could further improve the controller's accuracy.

It is also important to note that the cascaded system had trouble maintaining the highest possible jacket inlet temperature at a constant value, as can be seen in Figure 6(b). As this was the saturated upper value for the input, it is likely that the oscillations seen in the figure are a result of increased valve sensitivity in this area, where the hot water valve is fully open while the cold water valve is fully closed. Since the MPC is programmed with an upper input value constraint of $83^{\circ} \mathrm{C}$ (not $85^{\circ} \mathrm{C}$; this is explained in the next section), in the case when the pure hot water temperature rises slightly above the constraint (i.e., $83.4^{\circ} \mathrm{C}$ ), the cascaded control system minimally opens the cold valve to lower the temperature to the desired $83^{\circ} \mathrm{C}$. Because the 


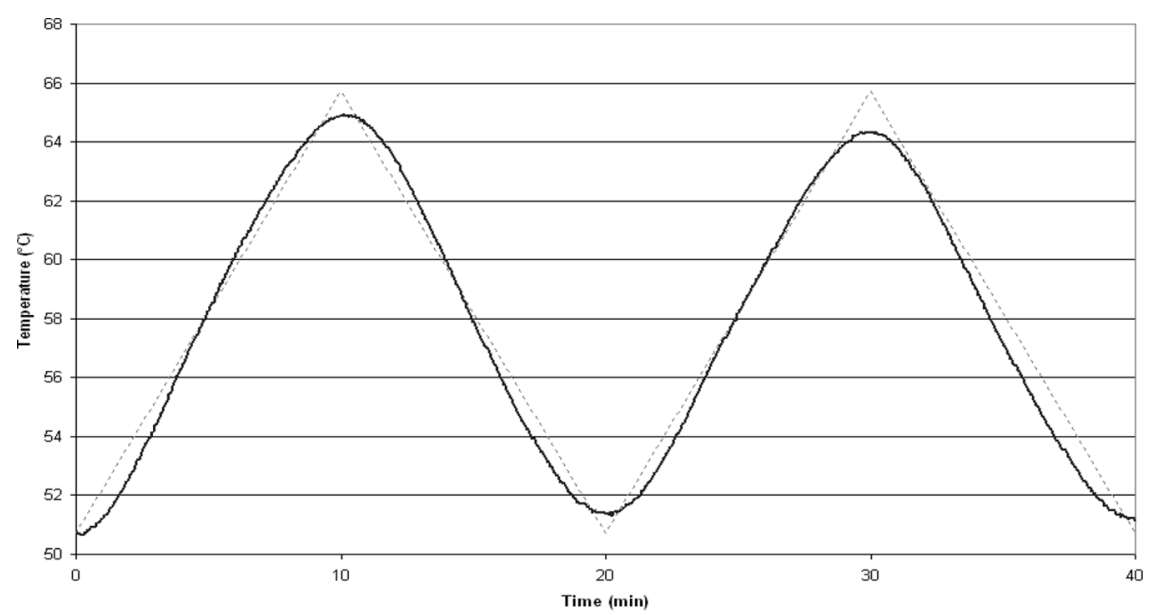

(a)

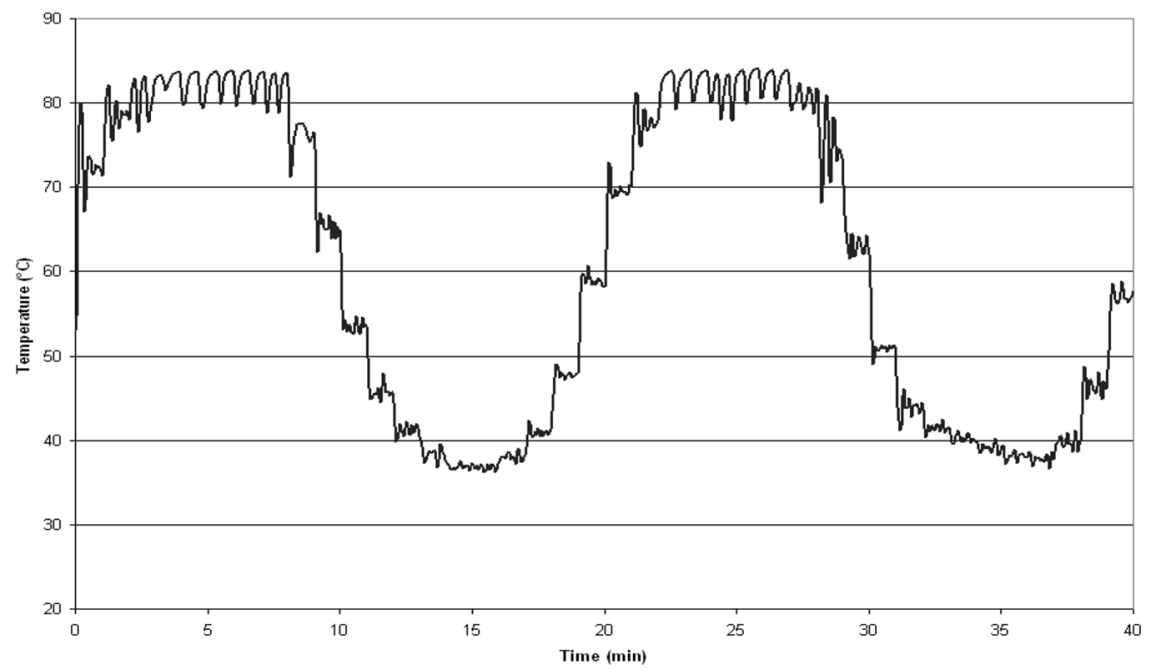

(b)

Figure 6. Experimental MPC tracking of the ramp for $\tau_{p}=20 \mathrm{~min}$, with the thinner, lighter line representing the programmed set point trajectory and the darker line representing actual performance in (a). An average error of $0.543^{\circ} \mathrm{C} /$ reading was observed. The corresponding input (inlet jacket temperature) values are given in (b).

relationship between valve opening and flow rate is nonlinear, even a minimal opening tends to send too much cold water, resulting in a higher decrease in temperature. This is the likely reason for the oscillations seen in the input values of the upper boundary. While further work to correct this problem is forthcoming, it is not believed that it significantly harms the MPC controller's performance.

In the next subsection, dynamic operability studies are discussed, performed in order to verify the inherent limitations of the current process design and to propose an increase in the jacket inlet flow rate, thus enabling the system to achieve even steeper ramps. 


\section{Dynamic Operability Analysis and Results}

The discussion in the previous section dealt with the improvement of the controller's accuracy in following a ramp set point but did not address the concepts of speed and feasibility. For cases where $\tau_{p}=10 \mathrm{~min}$, both in the experiments and in the simulations, the MPC control system qualitatively "fails" to achieve the set point trajectory, coming several degrees short of the top and bottom points. In the experimental case, this problem is worse, as the system either does poorly or fails entirely, the latter occurring because it is unable to find an optimized solution within the preset constraints, a case that then results in no control action.

The inherent limitations of the system can be explored by applying dynamic operability analysis (Uztürk and Georgakis, 2002) to the scenario at hand, and they were studied here by first analyzing the simulations and then actual experimental cases. The following equation (Uztürk, 2001) can be used to obtain the minimum time in which the temperature in the crystallizer could be changed from one value to another by changing the temperature of the jacket inlet:

$$
t_{m}=\tau \ln \left(\frac{\tilde{y}_{0}-K_{p} \tilde{u}^{*}}{\tilde{y}_{s e t}-K_{p} \tilde{u}^{*}}\right)
$$

where $K_{p}$ and $\tau$ denote the process gain and the time constant for the SISO system, $\tilde{y}_{0}$ denotes the starting output value (crystallizer temperature), $\tilde{y}_{\text {set }}$ denotes the output target value, and $\tilde{u}^{*}$ denotes the saturated input value, where the symbol $\sim$ signifies that the variables are deviations from their steady states. For the positively sloped ramp in the scenario discussed in this article, $\tilde{y}_{0}$ would be 0 (since the cycling started from a steady state), $\tilde{y}_{\text {set }}$ would be 15 , and $\tilde{u}^{*}$ would be 30.5 (assuming that the initial ramp involves heating). It is important to note that the positive $\tilde{u}^{*}$ for the real system was not 32.5 as it was in the simulations. This is due to the physical heat losses through the pipes; the maximum inlet jacket temperature achieved was at about $83^{\circ} \mathrm{C}$. If the direction of the ramp were reversed, and the system were cooled down $15^{\circ} \mathrm{C}, \tilde{y}_{0}$ would still be 0 , but $\tilde{y}_{\text {set }}$ would become $-15^{\circ} \mathrm{C}$, and $\tilde{u}^{*}$ could be defined as -32.5 (no significant heat losses are suffered by the coolant when it is close to room temperature).

For the standard jacket inlet flow rate of $1.8 \mathrm{~L} / \mathrm{min}$ employed in the experiments and simulations, a theoretical step response identification was carried out using the heat transfer model, with process gain and time constant values of $0.967^{\circ} \mathrm{C} /{ }^{\circ} \mathrm{C}$ and 12.14 min being obtained, respectively. Using Equation (11), a minimum time, $t_{m}$, of $8.6 \mathrm{~min}$ to only go up the ramp was calculated, theoretically proving that the $5 \mathrm{~min}$ ramp (corresponding to a $\tau_{p}$ of $10 \mathrm{~min}$ ) for this specific scenario was infeasible, and thus unachievable by the controller, as a control system cannot overcome the inherent operability limitations of the designed process.

The operability of the system could, however, be improved by increasing the flow rate, due to both lower residence times and better convective heat transfer. By once again using the heat transfer model from Equations (1)-(3), as well as the minimum time calculation from Equation (11), the operability of the system at the higher flow rates of $2.4 \mathrm{~L} / \mathrm{min}$ and $3.0 \mathrm{~L} / \mathrm{min}$ was calculated and graphed as shown in Figure 7. From this figure, it can be seen that increasing the flow rate by as much as two-thirds of its original value should lower the time needed to reach the set points. For example, the time to go up the $15^{\circ} \mathrm{C}$ ramp from the given steady state is shortened by $8.1 \%$ (from 8.6 to $7.9 \mathrm{~min}$ ). As mentioned previously, due to higher 


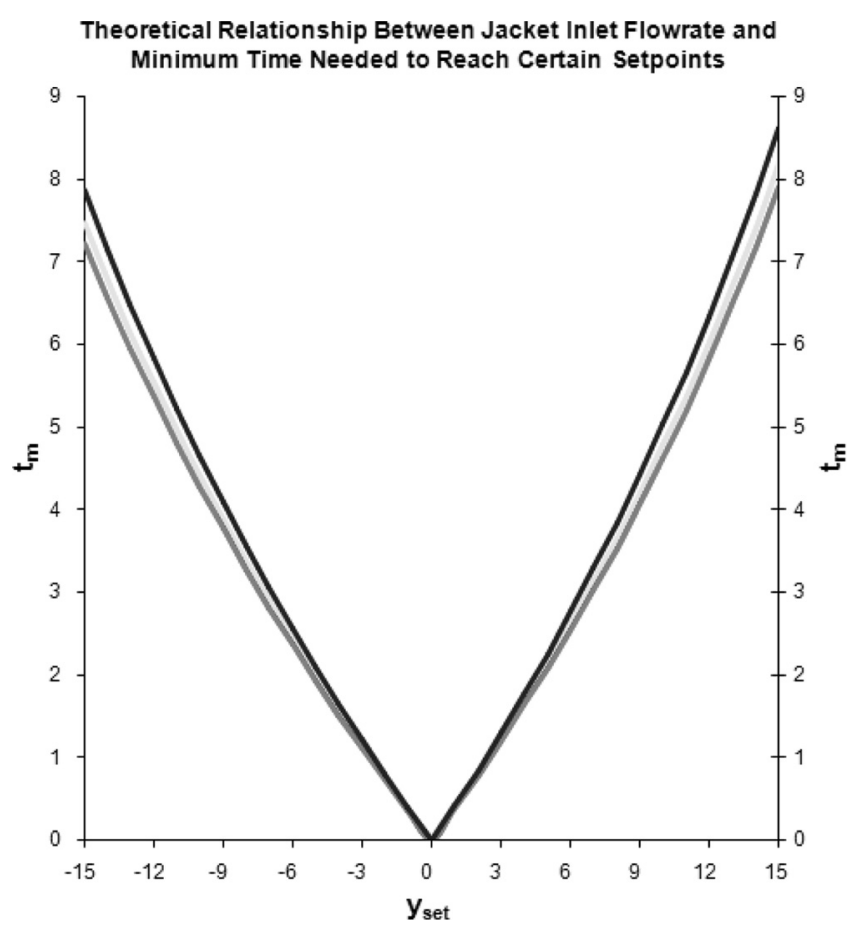

Figure 7. Theoretical analysis of the system's operability by analyzing how changes in the jacket inlet flow rate can change the minimum time $\left(t_{m}\right)$ needed to reach certain set points $\left(y_{\text {set }}\right)$. The lines, from top to bottom, represent flow rates of $1.8 \mathrm{~L} / \mathrm{min}, 2.4 \mathrm{~L} / \mathrm{min}$, and $3.0 \mathrm{~L} / \mathrm{min}$.

control power for the negatively sloped case, it can also be seen that less time is needed to lower the temperature than is needed to increase it.

Applying the same approach for two experimental scenarios (one at a flow rate of $1.8 \mathrm{~L} / \mathrm{min}$ and one at a flow rate of $2.4 \mathrm{~L} / \mathrm{min}$ ) confirmed that there was significant room for improvement experimentally, with the results given in Figure 8 . The experimental step response data $\left(K_{p}=0.916^{\circ} \mathrm{C} /{ }^{\circ} \mathrm{C}\right.$ and $\left.\tau=11.75 \mathrm{~min}\right)$ revealed that the system's response was slightly slower at a flow rate of $1.8 \mathrm{~L} / \mathrm{min}$ than was predicted by the model $\left(K_{p}=0.967^{\circ} \mathrm{C} /{ }^{\circ} \mathrm{C}\right.$ and $\left.\tau=12.14 \mathrm{~min}\right)$, while the speed of the system's response was about the same between the two for a flow rate of $2.4 \mathrm{~L} / \mathrm{min}\left(K_{p}=0.913{ }^{\circ} \mathrm{C} /{ }^{\circ} \mathrm{C}\right.$ and $\tau=10.55 \mathrm{~min}$ for experimental, $K_{p}=0.975^{\circ} \mathrm{C} /{ }^{\circ} \mathrm{C}$ and $\tau=11.65 \mathrm{~min}$ for theoretical) despite slightly differing parameters. As such, the potential improvement of increasing the flow rate by a third of its original value to $2.4 \mathrm{~L} / \mathrm{min}$ based on experimental results $(0.9 \mathrm{~min}, 10.0 \%)$ turned out to be significantly greater than the improvement based on theoretical studies $(0.4 \mathrm{~min}, 5.2 \%)$ for the rising $15^{\circ} \mathrm{C}$ ramp.

To follow up on these results, two operability scenarios were tested, an inoperable and an operable case, for two sets of operating conditions (a total of four runs). In the first set, the crystallizer temperature started at a steady state, climbed a $15^{\circ} \mathrm{C}$ ramp in either 6 (inoperable) or 9 (operable) min, and then remained constant at the higher temperature, with a jacket inlet flow rate of $1.8 \mathrm{~L} / \mathrm{min}$. In the second set, the same trajectories were used, but an optimized inlet flow rate was continuously 


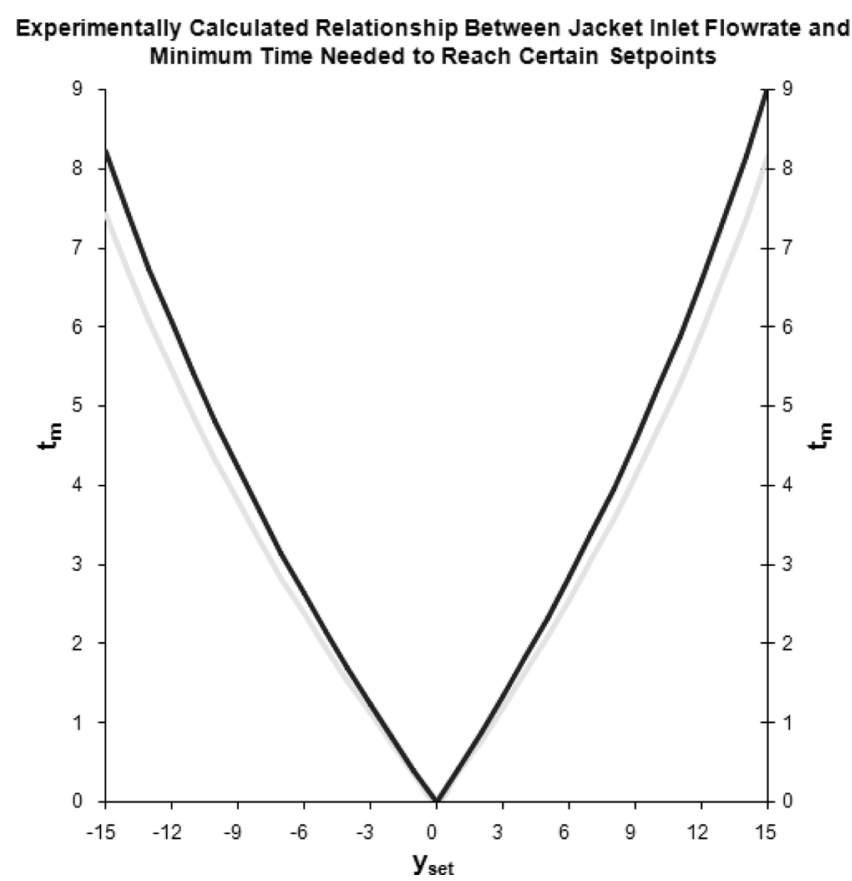

Figure 8. Effect of increased jacket inlet flow rate on the minimum time $\left(t_{m}\right)$ needed to reach certain set points $\left(y_{\text {set }}\right)$, as calculated from experimental step response data. The top line represents a flow rate of $1.8 \mathrm{~L} / \mathrm{min}$, with the bottom representing $2.4 \mathrm{~L} / \mathrm{min}$.

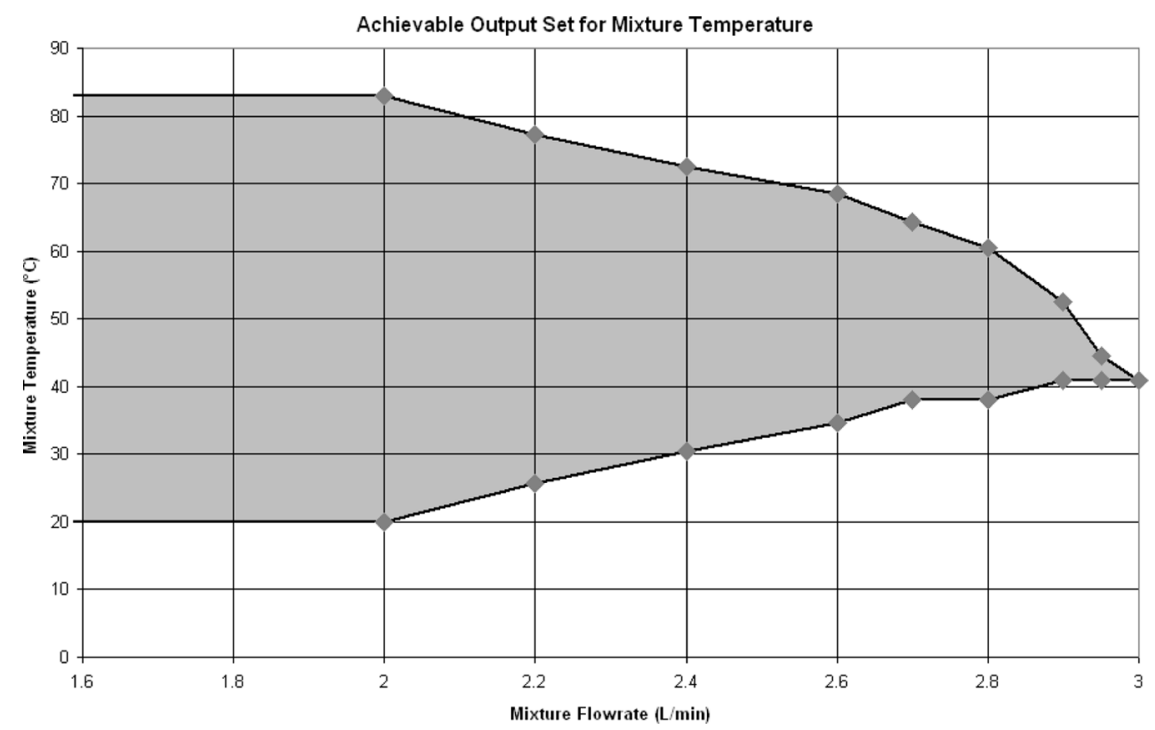

Figure 9. The preferred achievable output set (AOS) for a total inlet flow rate range of $0-3.0 \mathrm{~L} / \mathrm{min}(1.6 \mathrm{~L} / \mathrm{min}+$ shown here) was experimentally determined, as shown by the large data points, and is presented as the shaded area. Linear interpolation was done in between the points. 
defined throughout the process, as specified by the achievable output set (AOS) in Figure 9, the AOS being defined as the achievable combinations of mixture flow rate and temperature using the flow rate ranges of the hot and cold streams in the available input set, or AIS. The results for the two sets of operating conditions are presented in Figures 10 and 11.

Due to varying pressures from the hot and cold flows, as well as the AIS limitations of each flow to $2.0 \mathrm{~L} / \mathrm{min}$ for safety reasons, not all temperatures were

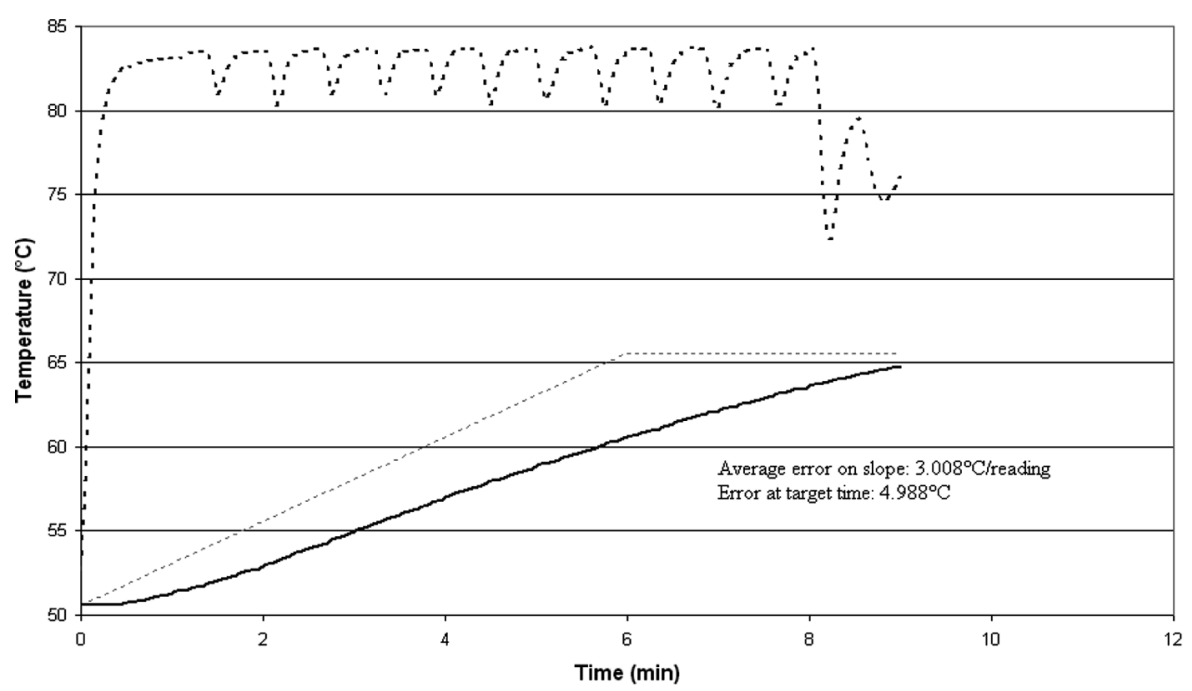

(a)

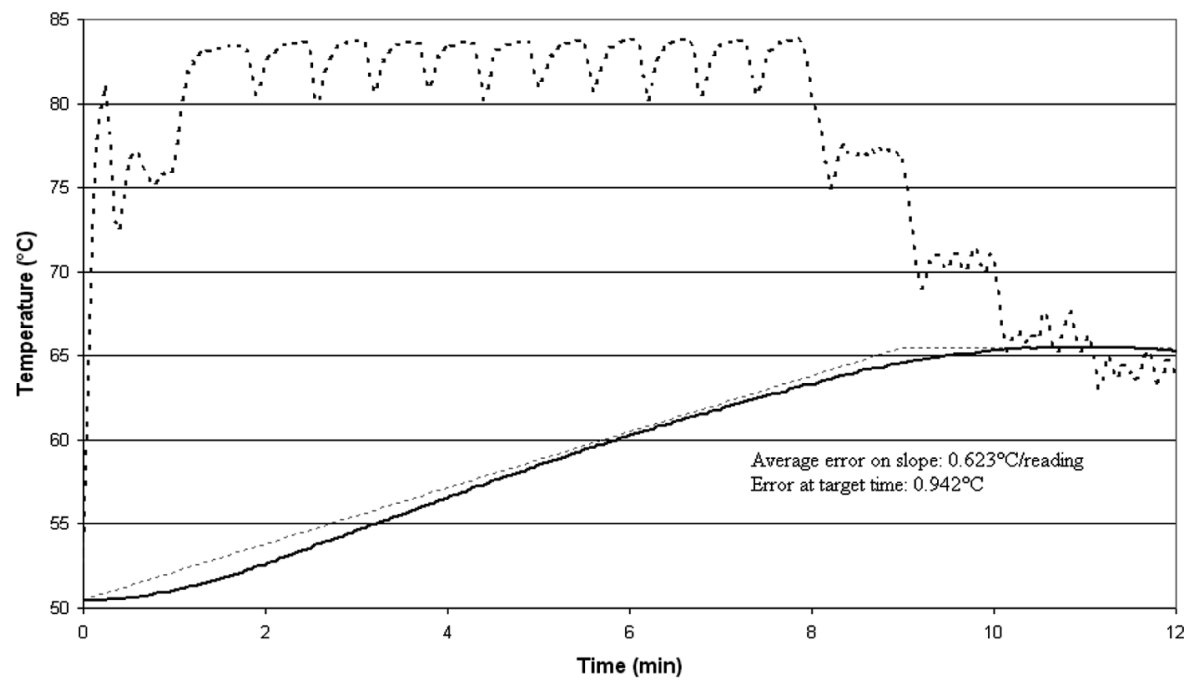

(b)

Figure 10. The system's performance for the inoperable (a) and operable (b) cases with a jacket inlet flow rate of $1.8 \mathrm{~L} / \mathrm{min}$, with the lighter dashed line denoting the set point trajectory and the solid line denoting actual performance. The darker dashed line denotes the corresponding input values. 


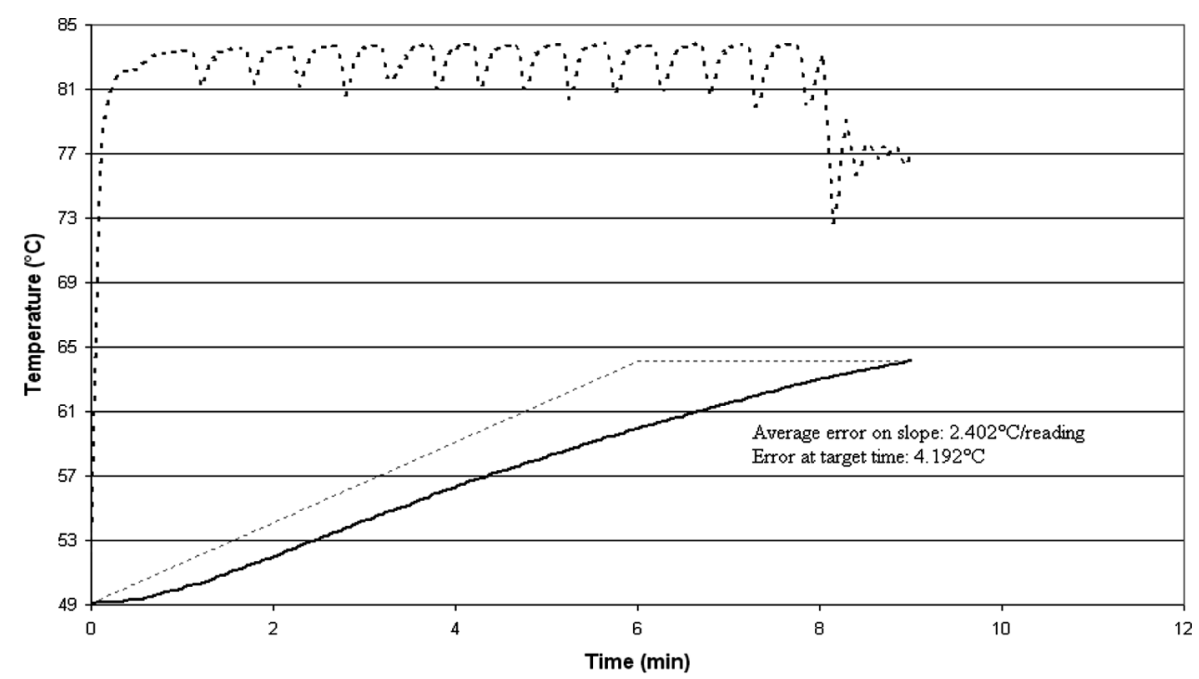

(a)

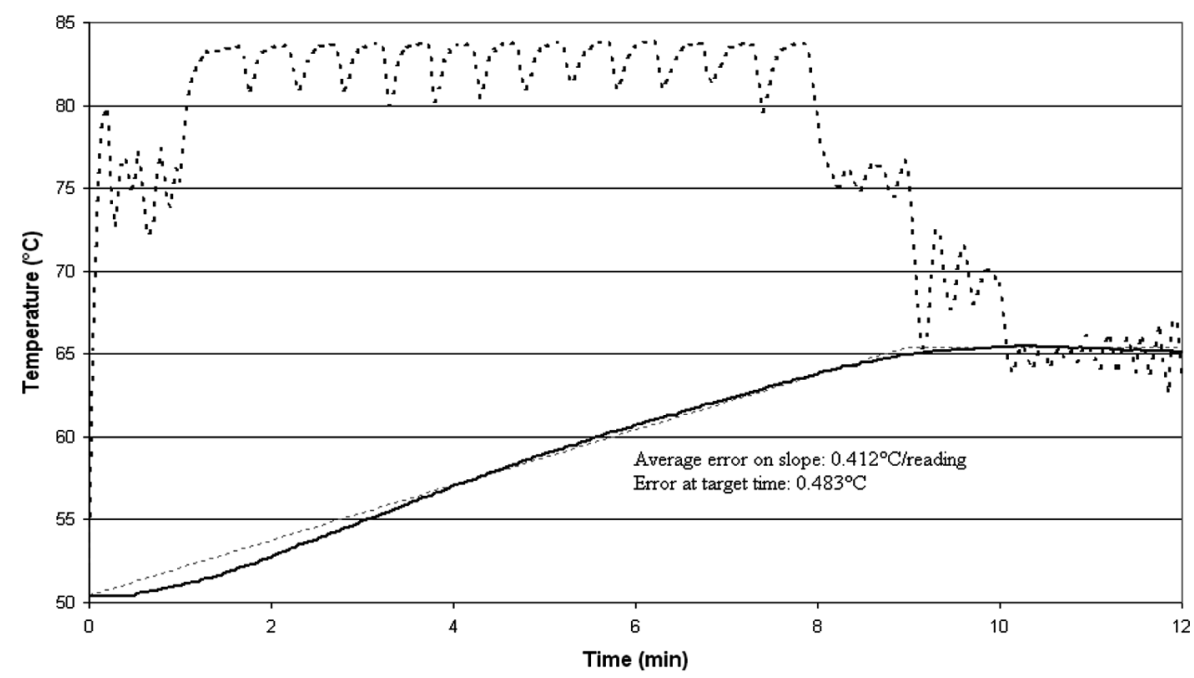

(b)

Figure 11. The system's performance for the inoperable (a) and operable (b) cases with the jacket inlet flow rate optimized between 2.0 and $3.0 \mathrm{~L} / \mathrm{min}$, with the lighter dashed line denoting the set point trajectory and the solid line denoting actual performance. The darker dashed line denotes the corresponding input values.

achievable for flows higher than $2.0 \mathrm{~L} / \mathrm{min}$ in the second set. The preferred AOS was therefore specified in the range of 2.0 to $3.0 \mathrm{~L} / \mathrm{min}$, where the entire temperature range was achievable at the lower boundary, but higher flow rates could be used for narrower temperature ranges. Experimentally, the flow rate was constantly varied from 2.0 to $3.0 \mathrm{~L} / \mathrm{min}$ depending on the range within which the MPC-specified inlet temperature fell, utilizing the maximum flow rate possible while achieving the desired inlet temperature. For example, in Figure 11(b), the first jacket inlet temperature that the MPC specified was at approximately $76^{\circ} \mathrm{C}$. In correspondence with the 
AOS, the maximum flow rate that the system could achieve this temperature at was about $2.2 \mathrm{~L} / \mathrm{min}$, and so this flow rate was used. When the MPC specified a new inlet temperature at the next sampling time one minute later (the saturated input value of $83^{\circ} \mathrm{C}$ ), the system referenced the AOS again and used the highest flow possible, which, for the saturated value, is $2.0 \mathrm{~L} / \mathrm{min}$.

As expected, the use of higher flow rates improved the operability of the system, and the error between the set point and the actual temperature along the ramp was cut significantly ( $\sim 33 \%$ reduction for the operable scenario and $\sim 20 \%$ reduction for the inoperable). It is important to note that the model employed for the higher flow runs was still the same linear model as before, derived for a flow rate of $1.8 \mathrm{~L} / \mathrm{min}$. A proper use of multiple linear models for varying flow rates should further improve the accuracy of the controller. Additionally, the safety pressure limits of the inlet flow can be studied further, allowing for possible expansion of the available input set, and thus the expansion of the achievable and desired output sets. Subsequent work will address these implementations.

Although the experimental runs discussed above were done only for a single ramp scenario, it is expected that they will be fully applicable to the cycling case. This is because they have been carried out for the more difficult side of the cycle (with less control power, as demonstrated in Figures 7 and 8), where the $\tilde{u}^{*}$ starts at $30.5^{\circ} \mathrm{C}$ and decreases to $15.5^{\circ} \mathrm{C}$ along the course of the run. In going back down $15^{\circ} \mathrm{C}$, $\tilde{u}^{*}$ would start at an absolute value of $47.5^{\circ} \mathrm{C}$ and would decrease down to only $32.5^{\circ} \mathrm{C}$ along the course of the run. Therefore, the promising results found here for the positively sloped ramp should carry over (with additional benefits) to the negatively sloped case.

\section{Conclusions}

The design, construction, and operation of a stirred tank with fast and accurate temperature control has been described in this article. With an ultimate goal of being able to use the operating conditions in the system to manipulate crystal shape, a cycling ramp temperature profile was chosen as the fundamental test of the controller's efficacy. After demonstrating that a traditional feedback controller was unlikely to fare well for steeper ramps, an MPC controller was implemented and achieved the desired trajectory reasonably well in practice, even for a temperature cycle of $15^{\circ} \mathrm{C}$ for a $20 \mathrm{~min}$ period. To improve the operability of, and to increase the range of feasible ramps for, the system, the use of higher jacket inlet flow rates was experimented with, and it was found that faster ramps were achievable if higher flows were used. By improving the dynamic operability of the system, it will be possible to push the limits of the controller even further.

This study has provided a starting point for the setup of a complete crystallization system with an advanced control scheme for crystal size and shape control. The next step is to equip the physical system with the probes necessary for concentration and crystal size measurements and to design a second model-based controller to control crystal properties by more specific temperature profiles passed on as set points to the controller discussed in this article. Once this is accomplished, experiments with varying crystallization conditions will be feasible, making it possible to design a process where the shape of a crystal could be controlled either by growing or dissolving in cycles, by optimal cooling and heating rates, or by a mixture of both. 


\section{Acknowledgments}

The authors would like to acknowledge Haris Siampanis for his aid in the construction of the crystallizer. Further acknowledgment is due to Sze Wing Wong and Praveen Prasanna, for their help in the theoretical crystallization background of this project.

\section{References}

Belanger, P. W., and Luyben, W. L. (1997). Design of low-frequency compensators for improvement of plantwide regulatory performance, Ind. Eng. Chem. Res., 36(12), 5339-5347.

Bequette, B. W., Holihan, S., and Bacher, S. (2004). Automation and control issues in the design of a pharmaceutical pilot plant, Control Eng. Pract., 12(7), 901-908.

Braatz, R. D. (2002). Advanced control of crystallization processes, Annu. Rev. Control, 26(1), 87-99.

Garcia, C. E., and Morshedi, A. M. (1986). Quadratic-programming solution of dynamic matrix control (QDMC), Chem. Eng. Commun., 46(1-3), 73-87.

Lundström, P., Lee, J. H., Morari, M., and Skogestad, S. (1995). Limitations of dynamic matrix control, Comput. Chem. Eng., 19(4), 409-421.

Miller, S. M., and Rawlings, J. B. (1994). Model identification and control strategies for batch cooling crystallizers, AIChE J., 40(8), 1312-1327.

Qin, S. J., and Badgwell, T. A. (2003). A survey of industrial model predictive control technology, Control Eng. Pract., 11(7), 733-764.

Shangfeng, Y., Genbo, S., Zhengdong, L., and Rihong, J. (1999). Rapid growth of $\mathrm{KH}_{2} \mathrm{PO}_{4}$ crystals in aqueous solution with additives, J. Cryst. Growth, 197(1-2), 383-387.

Shen, J. X., Chiu, M. S., and Wang, Q. G. (1999). A comparative study of model-based control techniques for batch crystallization process, J. Chem. Eng. Jpn., 32(4), 456-464.

Snyder, R. C., and Doherty, M. F. (2007). Faceted crystal shape evolution during dissolution or growth, AIChE J., 53, 1337-1348.

Uztürk, D. (2001). Dynamic Operability of Processes, 114-115, Lehigh University, Bethlehem, Penn.

Uztürk, D., and Georgakis, C. (2002). Inherent dynamic operability of processes: General definitions and analysis of SISO cases, Ind. Eng. Chem. Res., 41(3), 421-432.

Yang, G., Kubota, N., Sha, Z., Louhi-Kultanen, M., and Wang, J. (2006). Crystal shape control by manipulating supersaturation in batch cooling crystallization, Cryst. Growth Des., 6(12), 2799-2803.

Zhang, Y., Sizemore, J., and Doherty, M. F. (2006). Shape evolution of 3-dimensional faceted crystals, AICHE J., 52(5), 1906-1915.

Zhou, G. X., Fujiwara, M., Woo, X. Y., Rusli, E., Tung, H. H., Starbuck, C., Davidson, O., Ge, Z., and Braatz, R. D. (2006). Direct design of pharmaceutical antisolvent crystallization through concentration control, Cryst. Growth Des., 6(4), 892-898. 